\title{
Candidemia: características en los adultos mayores
}

\author{
Candidemia: characteristics in elderly patients
}

\author{
Natalia A. Díaz', Javier Farina ${ }^{1,2}$, Jorge Herrera ${ }^{3}$ y M. Cristina Ezcurra ${ }^{1}$
}

'Servicio de Infectología. Hospital Cesar Milstein. Buenos Aires, Argentina.

2Servicio de Infectología. Hospital Italiano de La Plata. Buenos Aires, Argentina.

${ }^{3}$ Servicio de Microbiología. Hospital Cesar Milstein. Buenos Aires, Argentina.

Javier Farina ha recibido honorarios como disertante de MSD y Pfizer. El resto de los autores no presenta conflictos de interés. No hubo fuente de financiamiento para este estudio.

Recibido: 15 de octubre de 2019 / Aceptado: 14 de abril de 2020

\section{Resumen}

Introducción: La candidemia es una micosis sistémica asociada a los cuidados de la salud de elevada morbimortalidad. Los adultos mayores presentan las tasas más altas y la información en esta población es escasa. Objetivos: Describir las características de las candidemias en los adultos mayores, identificar factores asociados a mortalidad y evaluar la utilidad de un score predictor. Métodos: Estudio retrospectivo de las candidemias en adultos mayores en un hospital de la ciudad de Buenos Aires. Se analizaron todos los episodios en pacientes mayores de 60 años en un período de ocho años. Resultados: Se incluyeron 59 episodios. La co-morbilidad más frecuente fue diabetes mellitus. El principal sitio de origen fue infección asociada a catéter (45\%). Sólo $17 \%$ presentó un Candida score mayor a 2,5. La mortalidad global a las $48 \mathrm{~h}$ fue $20 \%$ y a 15 días $49 \%$. Las variables asociadas a mortalidad fueron: shock, asistencia respiratoria mecánica (ARM), plaquetopenia, insuficiencia renal y leucocitosis (rango $p<0,0001-0,006)$. En el análisis multivariado el shock fue la única variable independiente asociada a mortalidad (HR 4,02 [IC 95\% 1,18-13,72], p=0,026). Conclusiones: La mitad de los adultos mayores con candidemia falleció en los primeros 15 días. Shock, ARM, plaquetopenia, insuficiencia renal y leucocitosis fueron factores asociados a mortalidad. El score predictor de uso habitual no fue útil en esta población.

Palabras clave: Candida spp; candidemia; adultos mayores; mortalidad; infección del torrente sanguíneo; infección asociada a catéter.

\begin{abstract}
Background: Candidemia is a nosocomial bloodstream infection and an important cause of comorbidity and mortality. Elderly patients present the highest rates of candidemia but data about this population is scarce. Aims: To describe characteristics of candidemia in elderly patients, to identify risk factors associated with mortality and to evaluate the usefulness of a predictive score. Methods: A retrospective observational study of candidemia in elderly patients from a tertiary care hospital was carried out. We analyzed all Candida bloodstream infections during an eight-year period in patients older than 60 years. Results: Fifty-nine episodes were included. Diabetes was the most frequent comorbidity. Most candidemia were catheter related infections (45\%). Only $17 \%$ of patients had a Candida score $>2.5$. Overall mortality at 48 hours was $20 \%$, and at 15 days was $49 \%$. Mortalityrelated factors were shock, assisted mechanical ventilation, thrombocytopenia, renal failure and leukocytosis ( $p$ range $<0.0001-0.006$ ). On multivariate analysis septic shock was independently associated with mortality (HR 4.02 [CI 95\% 1.18-13.72] p = 0.026). Conclusions: Nearly half of patients with candidemia died during the first fifteen days. Shock, assisted mechanical ventilation, thrombocytopenia, renal failure, and leukocytosis were factors associated with mortality. The predictive score was not useful in this population.

Keywords: Candida spp; candidemia; elderly; mortality; bloodstream infection; catheter related infection.
\end{abstract}




\section{Definiciones}

Candidemia: aislamiento, en al menos un hemocultivo as infecciones sistémicas por Candida spp. son una de las principales causas de infecciones del torrente sanguíneo asociadas a los cuidados de la salud y su incidencia se ha incrementado en los últimos años. Se caracterizan por su elevada morbilidad, prolongan la estadía hospitalaria e incrementan los costos del sistema sanitario ${ }^{1,2}$.

A pesar de los avances en el diagnóstico y tratamiento antifúngico, el pronóstico no ha mejorado considerablemente y estudios recientes continúan reportando altas tasas de mortalidad global de alrededor de 30 a $48 \%$ en la población general ${ }^{3-5}$.

Los adultos mayores presentan las mayores tasas de candidemia. Esto se atribuye a diversos factores tales como la alta frecuencia de co-morbilidades, cambios fisiológicos relacionados con el envejecimiento y mayores tasas de colonización ${ }^{6-8}$.

Si bien la candidemia en la población general ha sido bien estudiada, no ha recibido demasiada atención en los adultos mayores y las publicaciones son poco numerosas.

Resulta fundamental comprender ciertos aspectos de la candidemia en esta población, como la presentación clínica, la utilidad de los scores predictores, los factores de riesgo y tener un elevado nivel de sospecha para iniciar un tratamiento oportuno y adecuado, a fin de disminuir la mortalidad.

\section{Objetivos}

Describir las características clínicas y microbiológicas de los pacientes con candidemia, identificar los factores asociados a mortalidad y evaluar la utilidad de un score predictor en los adultos mayores.

\section{Métodos}

Estudio observacional, retrospectivo, de las candidemias en pacientes mayores de 60 años del Hospital César Milstein, institución de tercer nivel, especializada en adultos mayores de la ciudad de Buenos Aires, Argentina.

Se incluyeron todos los pacientes hospitalizados que presentaron al menos un episodio de candidemia en el período de ocho años y medio, comprendido entre el 1 de enero de 2011 y 31 de junio de 2019.

Se describen las características demográficas, comorbilidades, características de la hospitalización, aislamientos microbiológicos, el score predictor de candidemia Candida Score $e^{9}$ y el score de co-morbilidades de Charlson (Charlson Comorbidity Index $)^{10}$.

Los datos se obtuvieron de las historias clínicas y registros informatizados de los pacientes. tomado por venopunción periférica, de cualquier especie de Candida.

Infección asociada a catéter: aislamiento microbiológico de cualquier especie de Candida en cultivo semicuantitativo de punta de catéter con recuento $\geq 15$ unidades formadoras de colonias con la técnica de $\mathrm{Maki}^{11} \mathrm{o} \geq 10^{3}$ con la técnica de Brun-Buisson ${ }^{12}$ o en hemocultivos tomados a través del catéter, más aislamiento simultáneo de la misma especie de Candida en hemocultivos tomados por venopunción. En el caso de los cultivos tomados a través del catéter se tuvo en cuenta para el diagnóstico, hemocultivos tomados a través del catéter con respecto a los hemocultivos tomados por venopunción ${ }^{13}$. En los casos en los que no hubo tiempo diferencial $\geq 120 \mathrm{~min}$, si el paciente no presentaba otro foco de origen probable de la candidemia, se consideró también como infección asociada a catéter.

Candidemia primaria: aislamiento de cualquier especie de Candida en hemocultivos sin evidencia del foco de origen.

Se midió la mortalidad, definida como muerte por todas las causas a las $48 \mathrm{~h}$, a los 15 y 30 días desde el momento del diagnóstico de candidemia y los factores de riesgo relacionados.

\section{Estudios microbiológicos}

Para el estudio de los hemocultivos se utilizó el BacT/ ALERT ${ }^{\circledR} 3 \mathrm{D}$ (bioMérieux). Los hemocultivos fueron incubados por 7 días. Para la identificación microbiológica, entre 2011 y 2014 se utilizaron agar cromogénico (CHROMagar Candida ${ }^{\circledR}$ ) y API20C AUX ${ }^{\circledR}$ (bioMérieux). A partir de 2014, también se dispone de VITEK 2 System ${ }^{\circledR}$ (bioMérieux) para identificación de especie. La sensibilidad antimicrobiana in vitro se realizó por métodos manuales (discos de antimicrobianos, E-test ${ }^{\mathbb{}}$ ) y en algunas cepas por ATB FUNGUS 3 (bioMérieux) y VITEK 2 System $^{\circledR}$ (bioMérieux) e interpretados según los estándares del CLSI (Clinical Laboratory Standards Institute).

\section{Análisis estadístico}

Se utilizó el test de $\chi^{2}$ para el análisis bivariado de las variables categóricas y Mann Whitney para el análisis de las variables continuas con distribución de datos no paramétrica. Se realizó un modelo multivariado de riesgos proporcionales de Cox para comparar el tiempo a la muerte como variable dependiente, usando como predictores las variables que tuvieron una significancia estadística en el análisis bivariado. Se realizó una curva de Kaplan Meier para mostrar el efecto del tiempo en la mortalidad de los pacientes con candidemia. Se consideró significativa una $p<0,05$. el tiempo diferencial de positivización $\geq 120$ min de los 


\section{Resultados}

\section{Episodios totales y tasas de incidencia}

Entre enero de 2011 y junio de 2019 se diagnosticaron 63 episodios de candidemia en 61 pacientes, representando una incidencia de 1,25\% sobre el total de admisiones y $23,9 \%$ sobre las admisiones en terapia intensiva. De estos episodios, 59 fueron en mayores de 60 años, los que fueron incluidos en el análisis.

\section{Características de los pacientes}

La mediana de la edad fue de 74 años (rango intercuartil [RIC] 67-81 años) y 56\% fueron mujeres. El score de Charlson promedio fue de 4,5 y el Karnofsky 44.

Las co-morbilidades más frecuentes fueron: diabetes mellitus $(30,5 \%)$, insuficiencia renal crónica $(27 \%)$ $\mathrm{y}$ tumores sólidos $(27 \%)$. Siguiendo en orden de fre-

Tabla 1. Características de 59 pacientes mayores de 60 años con candidemia y sus factores predisponentes

\section{n (\%)}

Mediana (RIC)

Características de los pacientes

$\begin{array}{ll}\text { Mujeres } & 33(56) \\ \text { Edad (años) } & 74(67-81) \\ \text { Diabetes mellitus } & 18(30,5) \\ \text { IRC } & 16(27) \\ \text { Tumores sólidos } & 16(27) \\ \text { ICC } & 11(18,6) \\ \text { EPOC } & 7(11,8) \\ \text { Neoplasias hematológicas } & 4(6,7) \\ \text { Enfermedades autoinmunes } & 4(6,7) \\ \text { Cirrosis hepática } & 1(1,7)\end{array}$

Factores predisponentes

$\begin{array}{lc}\text { CVC } & 48(81) \\ \text { ARM } & 18(30) \\ \text { Catéter vesical } & 42(71) \\ \text { Total de cirugías } & 26(44) \\ \text { Cirugías traumatológicas } & 14(54) \\ \text { Cirugías abdominales } & 12(46) \\ \text { ATB previo } & 52(88) \\ \text { Uso de corticosteroides } & 7(11,8) \\ \text { Neutropenia } & 2(3,4) \\ \text { Nutrición parenteral } & 2(3,4)\end{array}$

IRC: insuficiencia renal crónica; ICC: insuficiencia cardíaca congestiva; EPOC: enfermedad pulmonar obstructiva crónica; CVC: catéter venoso central; ARM: asistencia respiratoria mecánica; ATB: antibacteriano cuencia, insuficiencia cardíaca, EPOC, uso crónico de corticosteroides, neoplasias hematológicas, enfermedades autoinmunes, úlcera gástrica perforada y cirrosis. Sólo dos pacientes recibían nutrición parenteral al momento de la candidemia (Tabla 1).

En $44 \%$ de los episodios, los pacientes habían sido sometidos a una cirugía mayor durante la misma internación, (54\% cirugías traumatológicas y 46\% cirugías abdominales).

La mediana de días de internación previo al evento fue de 26 días (RIC 15-47).

Del total de episodios de candidemia, 37\% se registraron en terapia intensiva. La mediana de días de internación en la unidad previo al evento fue 13 días (RIC 11-28).

Cincuenta y dos $(88 \%)$ pacientes habían recibido terapia antibacteriana en el último mes, con un promedio de tres antimicrobianos distintos, de los cuales $71 \%$ fueron carbapenems.

\section{Presentación clínica y scores predictores}

El 44\% de los episodios se presentó sólo con fiebre y $39 \%$ con shock séptico.

Al analizar scores predictores, sólo 17\% presentó un Candida Score mayor a 2,5 el día del evento.

\section{Características microbiológicas}

Del total de cepas aisladas, 22 (37,2\%) aislados fueron C. albicans; 12 (20,3\%) C. tropicalis; 10 (16,9\%) Candida spp.; 10 (16,9\%) C. parapsilosis; 4 (6,7\%) C. glabrata; 2 (3,3\%) C. guilliermondii. Hubo un solo paciente que presentó candidemia por dos especies distintas simultáneamente (Tabla 2).

El tiempo promedio de desarrollo en los hemocultivos fue de $47 \mathrm{~h}$. Sólo se obtuvo dos aislados resistentes a fluconazol (uno C. guilliermondii y otro C. tropicalis). Ninguno de los dos pacientes había estado expuesto previamente a azoles.

\section{Sitio de origen de la candidemia}

El sitio de origen más frecuente de la candidemia fue la infección asociada a catéter $(45,7 \%)$, seguido por candidemia primaria $(35,5 \%)$ y en tercer lugar pielonefritis.

\section{Complicaciones de la candidemia}

Se realizó ecocardiograma en búsqueda de vegetaciones en $42 \%$ de los pacientes, de quienes, dos presentaron endocarditis (8\%) y uno de ellos falleció a los 9 días. Fondo de ojos se realizó en $32 \%$ de los pacientes, sin hallazgos de impactos sépticos.

Se obtuvo hemocultivos de control en 40 (68\%) pacientes; hubo hemocultivos de control positivos en 10 (26\%), de los cuales falleció el 30\%. De los 19 pacientes a los que no se les realizó hemocultivos de control, 11 $(58 \%)$ habían fallecido antes de las primeras $48 \mathrm{~h}$ de iniciado el tratamiento. 


\begin{tabular}{|c|c|c|c|c|c|c|c|}
\hline \multirow[b]{2}{*}{ Focos de origen } & \multirow[b]{2}{*}{ n (\%) } & \multirow[b]{2}{*}{ C. albicans } & \multirow[b]{2}{*}{ C. tropicalis } & \multicolumn{2}{|c|}{ Especies de Candida } & \multirow[b]{2}{*}{ C. glabrata } & \multirow[b]{2}{*}{ C. guilliermondii } \\
\hline & & & & C. spp & C. parapsilosis & & \\
\hline IAC & $27(45,7)$ & $5 \quad(18)$ & $8(30)$ & $7(26)$ & $6(22)$ & & $2(7)$ \\
\hline Candidemia primaria & $21(35,5)$ & 11 (31) & $4(11)$ & $1(3)$ & $3(8)$ & $2(5)$ & \\
\hline Pielonefritis & $4(6,7)$ & $2(50)$ & & & & $2(50)$ & \\
\hline ISQ & $4(6,7)$ & $2(50)$ & & $1(25)$ & $1(25)$ & & \\
\hline Peritonitis & $2(3,3)$ & $1 \quad(50)$ & & $1(50)$ & & & \\
\hline \multirow[t]{2}{*}{ ITU } & $1(1,6)$ & $1(100)$ & & & & & \\
\hline & n total (\%) & $22 \quad(37)$ & $12(20)$ & $10(17)$ & $10(17)$ & $4(7)$ & $2(3)$ \\
\hline
\end{tabular}

\begin{tabular}{|c|c|c|c|c|}
\hline & $\begin{array}{c}\text { Pacientes vivos a } 30 \text { días } \\
\text { n (\%) }\end{array}$ & $\begin{array}{l}\text { Pacientes fallecidos a } 30 \text { días } \\
\text { n (\%) }\end{array}$ & OR (IC 95\%) & $p$ \\
\hline Shock & $2(8,7)$ & $21(91,3)$ & $43,7(3,79-504)$ & $<0,000$ \\
\hline ARM & $3(16,7)$ & $15(83,3)$ & $8,57(1,78-41,3)$ & 0,001 \\
\hline Infección en UTI & $5(22,7)$ & $17(77,3)$ & $5,34(1,45-19,73)$ & 0,004 \\
\hline Insuficiencia renal aguda & $4(20)$ & $16(80)$ & $6,13(1,71-21,93)$ & 0,005 \\
\hline Leucocitosis $\left(>12.000 / \mathrm{mm}^{3}\right)$ & $6(24)$ & $19(76)$ & $5,60(1,78-17,55)$ & 0,003 \\
\hline Plaquetopenia $\left(<120 \times 10^{3} / \mathrm{mm}^{3}\right)$ & $4(20)$ & $16(80)$ & $6 \quad(1,65-21,74)$ & 0,006 \\
\hline
\end{tabular}

\section{Tratamiento antifúngico}

Respecto al tratamiento antifúngico, $10(17 \%)$ de los pacientes no llegaron a recibirlo, ocho de ellos fallecieron en las primeras $48 \mathrm{~h}$. El retardo en el inicio del tratamiento tuvo un promedio de $45 \mathrm{~h}$ desde la toma de muestra.

De los pacientes cuya manifestación clínica fue el shock, en $52 \%$ el tratamiento antifúngico iniciado fue con equinocandinas y en 13\% fluconazol. En 35\% de los pacientes sin shock se inició tratamiento con equinocandinas y en $48 \%$ con fluconazol. No hubo diferencias estadísticamente significativas en cuanto al antifúngico de inicio elegido y mortalidad en pacientes con shock ( $\mathrm{p} 0,28)$, y sí hubo diferencia estadísticamente significativa a favor de las equinocandinas en pacientes sin shock (p 0,003).

El tiempo promedio de tratamiento, excluyendo los pacientes fallecidos en las primeras $48 \mathrm{~h}$ y los pacientes con endocarditis, fue de 12,5 días.

\section{Mortalidad}

La mortalidad total en las primeras $48 \mathrm{~h}$ fue de $20 \%$, a 15 días $49 \%$ y a 30 días $54 \%$ (Figura 1). La mediana del tiempo hasta la muerte fue de 6 días (RIC 2-9).

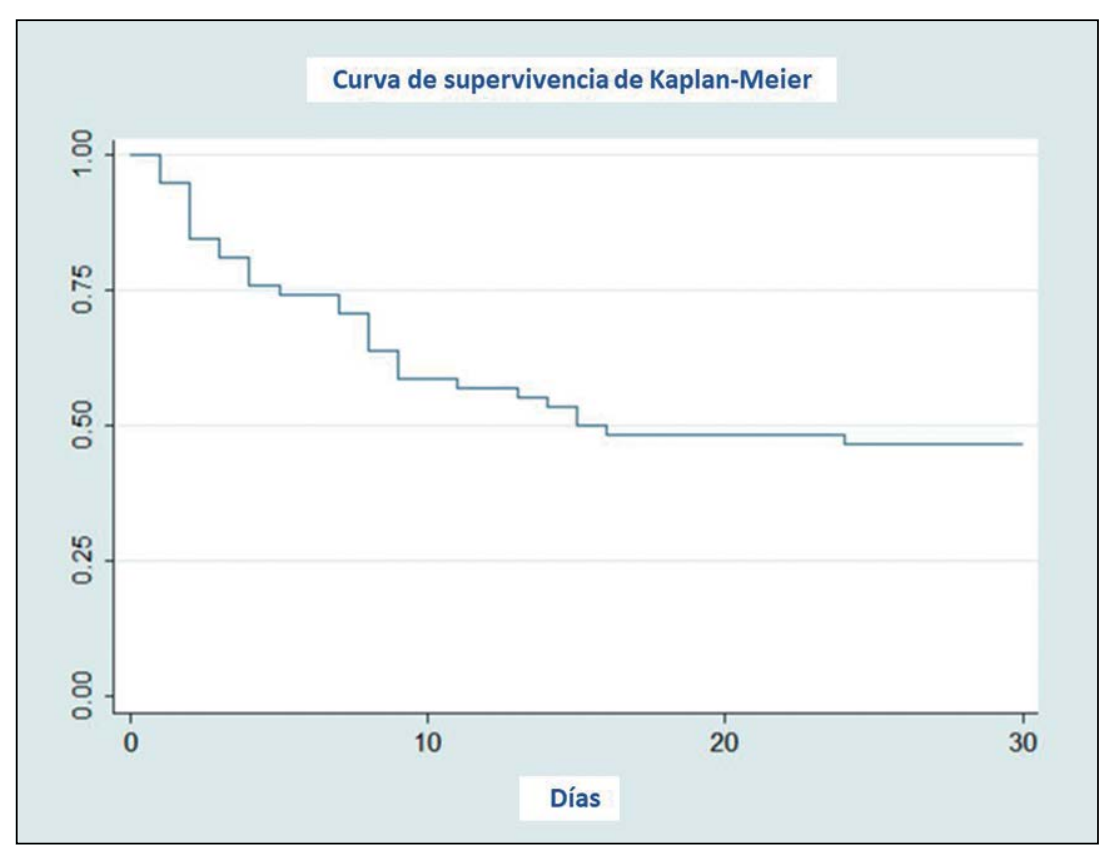

Figura 1. 
En el análisis bivariado, las principales variables asociadas a mortalidad a 30 días fueron: infección en terapia intensiva, deterioro de la función renal, leucocitosis, plaquetopenia, necesidad de asistencia respiratoria mecánica (ARM) y shock (Tabla 3).

En el análisis multivariado de riesgos proporcionales de Cox para comparar el tiempo a la muerte como variable dependiente, se utilizaron como predictores las variables que tuvieron una significancia estadística en el análisis bivariado: recuento de leucocitos y plaquetas, creatininemia, infección en terapia intensiva y ARM. La presencia de shock fue la única variable asociada a mortalidad (HR 4,02 [IC 95\% 1,18-13,72]; p 0,026).

\section{Discusión}

La bibliografía sobre candidemias en adultos mayores no es abundante, aunque en los últimos años se le ha dado mayor importancia, probablemente por el aumento de la población de adultos mayores y la relación de esta infección con los cuidados de la salud.

En el presente estudio observamos una elevada mortalidad de los adultos mayores con candidemia. Aproximadamente la mitad de los mayores de 60 años que padecieron una candidemia fallecieron en los primeros 15 días. Esta tasa de mortalidad es mayor que en la población general, pero se aproxima a lo reportado en otros estudios en este grupo etario ${ }^{7,8,14}$.

Los pacientes que presentaron una candidemia tuvieron internaciones muy prolongadas, con una media de 26 días previos al evento y la mayoría de ellos (88\%) experimentó una presión de selección por el uso previo de antibacterianos como un posible factor de riesgo.

A. Ramos Martínez y cols., analizaron de forma retrospectiva los datos de un estudio multicéntrico de candidemias, que incluyó 176 eventos en diferentes centros de España, y reportaron una mortalidad de $42 \%$ en la subpoblación de mayores de 75 años. A su vez, compararon las características de los eventos de candidemia en adultos mayores y adultos jóvenes. Encontraron que, en los adultos mayores, uno de los factores de riesgo de mortalidad fue la insuficiencia renal aguda, entre otros, lo que concuerda con nuestra serie. También observaron que el tratamiento antifúngico inadecuado, e incluso la falta de tratamiento, fueron más frecuentes en la población de adultos mayores. Fue notable la baja proporción de factores de riesgo "clásicos" de candidemia en este grupo etario, como la neutropenia y la nutrición parenteral, hecho que también observamos en nuestra serie, ya que hubo sólo dos pacientes neutropénicos y dos con nutrición parenteral que desarrollaron candidemia, lo que hace más difícil la sospecha diagnóstica y el inicio de tratamiento temprano ${ }^{8}$. Cabe destacar la falta de utilidad del score predictor de candidemia en nuestra población ya que sólo
$17 \%$ de nuestros pacientes con candidemia presentó un Candida score $>2,5^{9}$.

F. Barchiesi y cols., también reportaron una tasa de mortalidad a 30 días de $45 \%$ en la población de adultos mayores versus $28 \%$ en los adultos jóvenes y el shock séptico fue un factor de riesgo independiente de mortalidad en el primer grupo, lo que concuerda con nuestra serie ${ }^{7}$.

Con respecto a las especies aisladas, C. albicans sigue siendo la especie que más frecuentemente causa candidemia; sin embargo, últimamente se ha visto un aumento de las tasas de candidemia causadas por Candida noalbicans. En los últimos años se han reportado en distintos países múltiples brotes de $C$. auris, principalmente en unidades de cuidados intensivos, aunque en la Argentina aún no se han identificado casos hasta el momento de la redacción de este estudio ${ }^{15-18}$.

En nuestra institución, $C$. albicans fue la especie que originó el mayor número de candidemias, lo que coincide con los reportes epidemiológicos mundiales ${ }^{1,2,19}$. Le sigue en orden de frecuencia $C$. tropicalis, la segunda especie más frecuente en América Latina ${ }^{19}$, a diferencia de lo que ocurre en Europa donde la segunda especie más frecuente suele ser $C$. parapsilosi $s^{3,8,14}$. No se encontraron diferencias significativas en cuanto a las distintas especies de Candida y mortalidad, al igual que en la cohorte de A. Ramos Martínez y cols. ${ }^{8}$, ni tampoco entre los distintos focos de origen de la candidemia y mortalidad, aunque no se pueden sacar conclusiones definitivas en este sentido debido al reducido número de pacientes de nuestro estudio.

Resulta destacable la alta tasa de sensibilidad a azoles (97\%); sólo hubo dos aislados resistentes a fluconazol en más de ocho años.

Las equinocandinas siguen siendo de elección en el tratamiento antifúngico de inicio de la candidemia. Son fármacos fungicidas y efectivos frente a cepas con sensibilidad disminuida o resistentes a fluconazol como C. glabrata y C. krusei, aunque cuando el paciente se encuentra en condiciones de estabilidad hemodinámica y en centros con baja incidencia de resistencia a azoles, las guías actuales de tratamiento avalan el inicio con fluconazol $^{19,20}$.

Paradójicamente, en nuestra serie no se observaron diferencias estadísticamente significativas en cuanto al tratamiento de inicio y la mortalidad en pacientes con shock (p 0,28) y sí se observó menor mortalidad en pacientes sin shock e inicio de tratamiento antifúngico con equinocandinas (p 0,003). Probablemente, esto se deba a la elevada mortalidad que tuvieron los pacientes con shock séptico, quienes fallecieron, en su mayoría rápidamente, de manera independiente al tratamiento antifúngico recibido.

Hubo diez pacientes que no llegaron a recibir tratamiento antifúngico, de los cuales ocho fallecieron en las 


\section{Conclusiones}

en los hemocultivos. Esta situación es todavía frecuente en las series publicadas, tanto en adultos mayores como en pacientes jóvenes y es uno de los principales desafíos a resolver en la actualidad. Nuevas tecnologías asoman en estos últimos años con el objetivo de optimizar los tiempos de diagnóstico, como las basadas en reacción de polimerasa en cadena (RPC) multiple $\mathrm{x}^{21} \mathrm{o}$ en resonancia magnética $\mathrm{T} 2^{22}$.

Nuestro estudio presenta ciertas debilidades. Por un lado, al ser un estudio retrospectivo no pudimos obtener datos sobre las dosis de antifúngicos utilizadas ni sobre el retardo en el retiro de los catéteres en el caso de las infecciones asociadas a catéter. Hubo 10 aislados de Candida en que no se identificó la especie y fueron informadas como Candida spp. Por otro lado, es un estudio realizado en una única institución, por ende, contamos con un número reducido de pacientes que tal vez no nos permita evidenciar diferencias estadísticamente significativas en ciertos aspectos relevantes.
La candidemia es una infección invasora de elevada mortalidad en los adultos mayores. En muchas ocasiones es el evento final de una internación prolongada.

El score predictor de candidemia de uso habitual no fue útil en esta población. La falta de factores de riesgo clásicos en muchas situaciones dificulta el diagnóstico y, por ende, el tratamiento temprano, contribuyendo a elevar aún más las tasas de mortalidad.

Se requieren mayores esfuerzos para determinar con precisión los factores de riesgo asociados a esta entidad en los adultos mayores e identificar medidas que puedan ayudar a prevenir la candidemia con el objetivo de reducir la morbimortalidad que ocasiona.

Agradecimientos. A Diego Caruso y Fernando Di Fermo por el análisis estadístico.

\section{Referencias bibliográficas}

1.- Garnacho-Montero J, Díaz-Martín A, RuizPérez De Piappón M, García-Cabrera E. Infección fúngica invasiva en los pacientes ingresados en las áreas de críticos. Enferm Infecc Microbiol Clin 2012; 30 (6): 338-43. doi:10.1016/j.eimc.2012.02.011.

2.- Marchetti O, Bille J, Fluckiger U, Eggimann P, Ruef C, Garbino J, et al. Epidemiology of candidemia in Swiss tertiary care hospitals: secular trends, 1991-2000. Clin Infect Dis 2004; 38 (3): 311-20 doi:10.1086/380637.

3.- Puig-Asensio M, Padilla B, Garnacho-Montero J, Zaragoza O, Aguado J M, Zaragoza R, et al. Epidemiology and predictive factors for early and late mortality in Candida bloodstream infections: A population-based surveillance in Spain. Clin Microbiol Infect 2014; 20 (4): O245-54. doi: 10.1111/1469-0691.12380.

4.- Morgan J, Meltzer M I, Plikaytis B D, Sofair A N. Excess mortality, hospital stay, and cost due to candidemia: a case-control study using data from population-based candidemia surveillance. Infect Control Hosp Epidemiol 2005; 26 (6): 540-47. doi: 10.1086/502581

5.- Gudlaugsson O, Gillespie S, Lee K, Vande Berg J, Hu J, Messer S, et al. Attributable mortality of nosocomial candidemia, revisited. Clin Infect Dis 2003; 37 (9): 1172-7. doi: $10.1086 / 378745$.

6.- Wang H, Liu N, Yin M, Han H, Yue J, Zhang $\mathrm{F}$, et al. The epidemiology, antifungal use and risk factors of death in elderly patients with candidemia: A multicentre retrospective study. BMC Infect Dis 2014; 14 (1): 1-9. doi: 10.1186/ s12879-014-0609-x.

7.- Barchiesi F, Orsetti E, Mazzanti S, Trave F, Salvi A, Nitti C, et al. Candidemia in the elderly: What does it change? PLoS One. 2017; 12 (5): 1-14. doi:10.1371/journal. pone. 0176576 .

8.- Ramos-Martínez A, Vicente-López N, SánchezRomero I, Padilla B, Merino-Amador P, Garnacho-Montero J, et al. Epidemiology and prognosis of candidaemia in elderly patients. Mycoses 2017; 60 (12): 808-17. doi: 10.1111/ myc. 12677.

9.- León C, Ruiz-Santana S, Saavedra P, Nolla-Salas J, Álvarez-Lerma F, GarnachoMontero J, et al. A bedside scoring system ("Candida score") for early antifungal treatment in nonneutropenic critically ill patients with Candida colonization. Crit Care Med 2006; 34 (3): 730-7. doi: 10.1097/01. CCM.0000202208.37364.7D

10.- Charlson M E, Pompei P, Ales K L, MacKenzie C R. A new method of classifying prognostic comorbidity in longitudinal studies: Development and validation. J Chronic Dis 1987; 40 (5): 373-83. doi: https://doi.org/10.1016/00219681(87)901718.

11.- Maki D G, Weise C E, Sarafin H W. A semiquantitative culture method for identifying intravenous-catheter-related infection. N Engl J Med 1977; 296 (23): 1305-9. doi: 10.1056/ NEJM197706092962301.

12.- Brun Buisson C, Abrouk F, Legrand P, Huet Y, Larabi S, Rapin M. Diagnosis of central venous catheter-related sepsis: critical level of quantitative tip cultures. Arch Intern
Med 1987; 147 (5): 873-7. doi: 10.1001/ archinte.1987.00370050069012.

13.- Park K H, Lee M S, Lee S O, Choi S, Sung H, Kim M, et al. Diagnostic usefulness of differential time to positivity for catheterrelated candidemia. J Clin Microbiol 2014; 52 (7): 2566-72. doi: 10.1128/JCM.00605-14.

14.- Luzzati R, Cavinato S, Deiana M L, Rosin C, Maurel C, Borelli M. Epidemiology and outcome of nosocomial candidemia in elderly patients admitted prevalently in medical wards. Aging Clin Exp Res 2015; 27 (2): 131-7. doi: 10.1007/s40520-014-0251-x.

15.- Prakash A, Sharma C, Singh A, Kumar Singh P, Kumar A, Hagen F, et al. Evidence of genotypic diversity among Candida auris isolates by multilocus sequence typing, matrix-assisted laser desorption ionization time-of-flight mass spectrometry and amplified fragment length polymorphism. Clin Microbiol Infect. 2016; 22 (3): 277.e1-277.e9. doi: 10.1016/j. cmi.2015.10.022.

16.- Morales-López S E, Parra-Giraldo C M, Ceballos-Garzón A, Martínez H P, Rodríguez G J, Álvarez-Moreno C A, et al. Invasive infections with multidrug-resistant yeast Candida auris, Colombia. Emerg Infect Dis 2017; 23 (1): 162-4. doi: 10.3201/ eid2301.161497.

17.- Araúz A B, Caceres D H, Santiago E, Armstrong P, Arosemena S, Ramos C, et al. Isolation of Candida auris from 9 patients in Central America: Importance of accurate diagnosis and susceptibility testing. Mycoses. 2018; 61(1): 44-7. doi:10.1111/myc.12709.

18.- Calvo B, Melo A S A, Perozo-Mena A, 
Hernández M, Francisco E C, Hagen F, et al. First report of Candida auris in America: Clinical and microbiological aspects of 18 episodes of candidemia. J Infect 2016; 73 (4): 369-74. doi: 10.1016/j.jinf.2016.07.008.

19.- Nucci M, Thompson-Moya L, Guzman-Blanco M, Tiraboschi I N, Cortés J A, Echeverría J, et al. Recomendaciones para el manejo de la candidemia en adultos en América Latina. Rev Iberoam Micol 2013; 30 (3): 179-88. doi:10.1016/j.riam.2013.06.001.

20.- Pappas P G, Kauffman C A, Andes D R, Clancy C J, Marr K A, Ostrosky-Zeichner L, et al. Clinical Practice Guideline for the Management of Candidiasis: 2016 Update by the Infectious Diseases Society of America. Clin Infect Dis 2015; 62 (4): e1-e50. doi:10.1093/cid/civ933.

21.- Mylonakis E, Clancy C J, Ostrosky-Zeichner L, Garey K W, Alangaden G J, Vázquez JA, et al. T2 magnetic resonance assay for the rapid diagnosis of candidemia in whole blood: A clinical trial. Clin Infect Dis 2015; 60 (6): 8929. doi: 10.1093/cid/ciu959.

22.- Salimnia H, Fairfax M R, Lephart P R,

Schreckenberger P, DesJarlais S M, J. Johnson $\mathrm{J} K$, et al. Evaluation of the FilmArray blood culture identification panel: results of a multicenter controlled trial. J Clin Microbiol 2016; 54 (3): 687-98. doi:10.1128/JCM.0167915 . 